\title{
Bipolar Amplifier Bias Technique for Robust IM3 Null Tracking Independent of Internal Emitter Resistance
}

\author{
Toby Balsom \\ School of Engineering \\ The University of Waikato, NZ \\ Email: tcpb1@waikato.ac.nz
}

\author{
William Redman-White \\ Electronics and Computer Science \\ Southampton University, UK \\ Email:wrw@ecs.soton.ac.uk
}

\author{
Jonathan Scott \\ School of Engineering \\ The University of Waikato, NZ \\ Email: jbs@waikato.ac.nz
}

\begin{abstract}
Bipolar amplifiers can be biased to give a deep null in third order non-linearity, with the potential for high IP3 amplifier stages. This requires maintaining a precise voltage drop across a small resistive emitter degeneration resistance, whose value is related to $k T / q$. To make such a scheme practical, the bias must not only take into account the change in $k T / q$ with temperature, but must compensate for variations in the degeneration resistance. In this paper we present a bias technique for IM3 null tracking that can take account of temperature and resistance tolerances, and is also insensitive to the value of the internal emitter resistance. Simulations using a $27 \mathrm{GHz}$ BiCMOS technology indicate that the bias of an amplifier can be maintained over temperature, representative element tolerances, and mismatch such that the IP3 performance is maintained within $\pm 9.5 \mathrm{dBV}$ of the optimum null condition. The technique is applicable for a range of bipolar BiCMOS technologies and is attractive for amplifiers where high IP3 is required with moderate noise figure.
\end{abstract}

\section{INTRODUCTION}

The third-order null at a particular bias condition is a fundamental property of a degenerated BJT amplifier structure [1] [2] [3]. In principle it is possible to achieve very high IIP3 figures by the use of modest resistive degeneration and setting the bias such that the voltage across this resistance is $k T / 2 q$ [4] [5]. Such a configuration is potentially very attractive in applications where linearity is paramount while some noise figure can be sacrificed, such as in the RF mixer input in a radio receiver. However, to realise the theoretical benefits it is necessary to set the bias conditions with regard to the ambient temperatures so that the ideal degeneration voltage is maintained regardless of the temperature. This implies not only having a bias proportional to absolute temperature (PTAT), but also taking into account the temperature coefficient of the degeneration resistor itself. Both of these factors may be compensated for by means of a conventional PTAT bias and a matched resistance [6] [7]. However, the idea breaks down when the intrinsic device resistances are considered, since these cannot be easily accommodated in a conventional PTAT bias circuit. Indeed, the sensitivity of the third order null to the intrinsic emitter resistance is large enough that it can be used as a sensitive method for extracting the resistance value for a particular device layout [8]. The objective of this work is to develop a method for setting a BJT amplifier bias such that it operates at the third order null point over a wide temperature range, despite variations in the external degeneration resistance. Fig. 1 shows an IM3 null, and consequently an IIP3 peak, for a generic common-emitter amplifier. The null can be approximately modelled by (1) derived in [1] [4]

$$
I_{C}=\frac{k T}{2 q R_{e e}}
$$

where $I_{C}$ is the DC collector current, $k T / q$ is the thermal voltage and $R_{e e}$ is the summation of internal and external emitter resistance. This resistance should also contain the parasitic base resistance $R_{b}$ reflected through the beta of the transistor. Assuming $R_{b}$ is small and the beta is large, it can be approximated to just the internal and external emitter resistances. From this equation we see that any error in the effective value of $R_{e e}$ will cause the bias current to not be placed in the null. Hence, as (1) implies, we need to accurately extract the internal emitter resistance and use it with any external emitter resistance to bias a single transistor amplifier so as to maintain a voltage of $k T / 2 q$ across the total emitter resistance.

\section{TRANSLINEAR EXTRACTION}

A translinear stack circuit which allows cancellation of emitter resistances (sometimes broadly referred to as logarithmicconformance error) [6] [7], will form the basis which allows the design of a PTAT current source which is insensitive to internal emitter resistances. A simplified version of this is shown in Fig. 2a. This circuit relies on the current amplifier at the top, attempting to force equal currents through each transistor branch. The governing equation for a stack such as this is given by

$$
V_{R 1 a}=I_{1} R_{1 a}=\frac{k T}{q} \ln (A)-x I_{1} R_{E}
$$

where $R_{E}$ is the internal emitter resistance. This equation was simplified by taking scale factors $x$ and $A$ which are given by (3) and (4) below, where $M_{n}$ is the emitter area size for a 


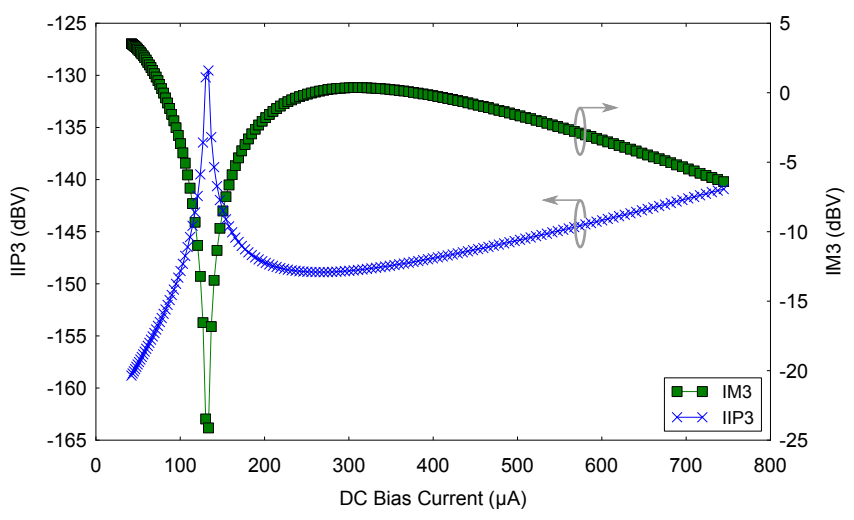

Fig. 1. Third order non-linearity null in a generic common-emitter amplifier using the $0.5 \mu \mathrm{m}$ BiCMOS model and chosen nominal circuit values.

given transistor.

$$
\begin{gathered}
x=\frac{1}{M_{4}}+\frac{1}{M_{5}}+\frac{1}{M_{6}}-\frac{1}{M_{1}}-\frac{1}{M_{2}}-\frac{1}{M_{3}} \\
A=\frac{M_{4} M_{5} M_{6}}{M_{1} M_{2} M_{3}}
\end{gathered}
$$

The first logarithmic term of (2) is an ideal PTAT component, but the last term $x I_{1} R_{E}$ is the portion of $V_{R 1 a}$ created by the devices' internal emitter resistance through the stack. It is possible to select conditions on scale factors $x$ and $A$ such that the contribution from the devices' internal emitter resistance is eliminated from $V_{R 1 a}$. This is done by observing the restrictions $\frac{1}{M_{4}}+\frac{1}{M_{5}}+\frac{1}{M_{6}}-\frac{1}{M_{1}}-\frac{1}{M_{2}}-\frac{1}{M_{3}}=0$ and $M_{4} M_{5} M_{6}>M_{1} M_{2} M_{3}$. We can express this condition as in (5) below, so that the value $V_{R 1 a}$ only contains the PTAT component.

$$
V_{R 1 a}=I_{1} R_{1 a}=\frac{k T}{q} \ln (A)
$$

It is thus possible to construct one complete translinear stack with the emitter resistance contribution completely removed (Fig. 2a) and a further similar stack where there is a contribution from a scaled portion of internal emitter resistance (Fig. 2b). The transistors in the first stack are dimensioned such that $x=0$ and while those in the second are set such that $x>0$, while both stacks use an equal scale factor for $A$. This creates two voltages, $V_{R 1 a}$ in the first stack and $V_{R 1 b}$ in the second stack, across the respective $R_{1}$ resistors which are temperature independent and contain known scaled portions of voltage contributed by internal emitter resistance. Because the two stacks must have different total parallel emitter areas to satisfy the required conditions, different equilibrium currents are set up in each stack. This prevents the extraction of the transistors internal emitter resistance by a simple subtraction of (2) and (5), but with more complex circuit design it is possible to extract the value of the devices as fabricated in each die.

\section{BIAS CURRENT EQUATION}

To produce the correct bias current we manipulate the two voltages $V_{R 1 a}$ and $V_{R 1 b}$. To express the required bias current

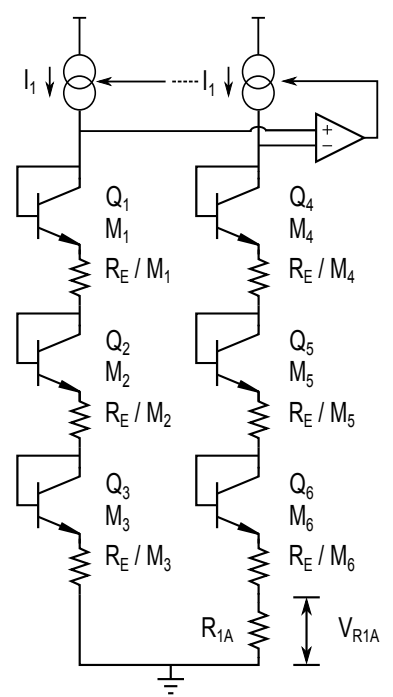

(a)

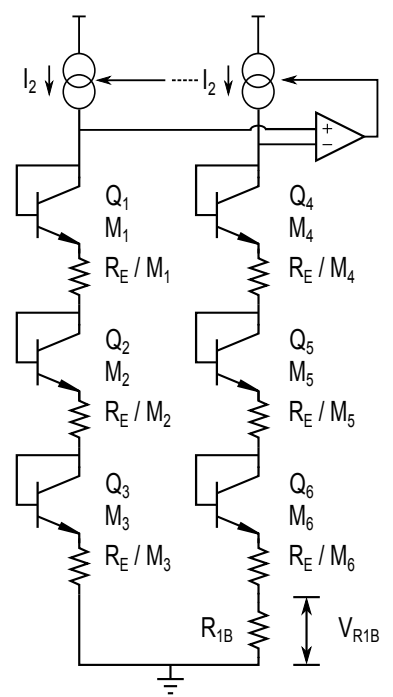

(b)
Fig. 2. Two translinear stack circuits, identical expect in BJT area ratios. Consequently a) uses $I_{1}$ to produce $V_{R 1 A}$ and b) uses $I_{2}$ to produce $V_{R 1 B}$.

mathematically, we define $V_{3}$ from the subtraction of the two voltages created from (2) and (5) as below.

$$
\begin{gathered}
V_{R 1 a}=I_{1} R_{1 a}=\frac{k T}{q} \ln (A) \\
V_{R 1 b}=I_{2} R_{1 b}=\frac{k T}{q} \ln (A)-x I_{2} R_{E} \\
V_{3}=V_{R 1 b}-V_{R 1 a}=x I_{2} R_{E}
\end{gathered}
$$

$I_{2}$ is the equilibrium current in the second stack with a nonzero $x$ value, so $V_{3}$ contains knowledge of $R_{E}$. Equation (1) implies that the target bias voltage will be $k T / 2 q$, and we seek a current that will drop this voltage across $R_{E}$ added to another resistor, $R_{1}$. We will achieve this using a reference current from which a portion is adjusted to allow for the $R_{E}$ contribution. $I_{1}$ is suitable for the reference as it contains no portion contributed by internal emitter resistance and is easily manipulated out of the first stack. From (6) we can obtain our desired reference bias current below in (9).

$$
I_{\text {Ref }}=\frac{k T}{2 q R_{1}}=\frac{I_{1}}{2 \ln (A)}
$$

It is also possible to rearrange (8), as below, to obtain $R_{E}$ on its own in terms of the known resistance $R_{1}$ (equal to $R_{1 a}$ and $R_{1 b}$ ) and the equilibrium currents of the two stacks, or as part of the total degeneration resistance $R_{E}+R_{1}$.

$$
\frac{R_{1}}{R_{E}+R_{1}}=\frac{x I_{2}}{x I_{2}+I_{1}-I_{2}}
$$

This creates a divider ratio for the internal and external emitter resistances. Combining this with the scale current in (9) we obtain our final equation for the bias current.

$$
I_{\text {Bias }}=\frac{I_{1}}{2 \ln (A)} \frac{x I_{2}}{x I_{2}+I_{1}-I_{2}}
$$




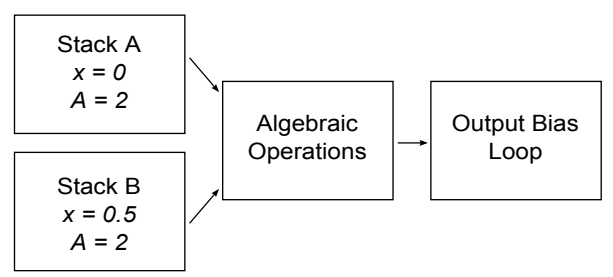

Fig. 3. Bias circuit blocks showing the four main stages of the circuit.

\section{Extraction Circuit Design}

The complete system has been designed and simulated using parameters from a commercial $0.5 \mu \mathrm{m} 27 \mathrm{GHz}$ BiCMOS process, typical for such RF applications, with a nominal 3.3V supply. Note that the system implementation is not specific to this technology, but the availability of NMOS and PMOS transistors is useful in the construction of the amplifiers and mirroring functions needed. The overall bias circuit consists of four main blocks as shown in Fig. 3. Firstly, the two translinear circuit stacks are used to extract two component voltages $V_{R 1 a}$ and $V_{R 1 b}$, and the related current values $I_{1}$ and $I_{2}$. These currents are passed to a third block which performs the required algebraic operations on the two voltages to produce an output scaled bias current. Finally, a bias loop uses the output to set the operating current in the signal path amplifier of interest $(\mathrm{Q} 1)$ so that the voltage across its total degeneration resistance is $k T / 2 q$ regardless of temperature and any variations in the values of $R_{E}$ or $R_{1}$. Current scaling, multiplication, and division circuit operations are required to obtain the mathematical result in (11). Current scaling can be done relatively easily with current mirrors and a multiply/divide operation can be achieved with a conventional translinear multiplier circuit which forms the majority of the second block. A servo current mirror loop can be used to build the driver block as seen in Fig. 5. The scale factors are chosen to be $A=2$ for both stacks, and $x=0, x=0.5$ for Stack A and $\mathrm{B}$ respectively. These stacks scale factors are accomplished with $M_{1-6}=2,2,2,1,4,4$ and $M_{1-6}=4,4,8,1,16,16$ for stacks A and B respectively. Numerical computations show that this combination is the smallest collective transistor array size which allows an $x=0.5$ scale factor in Stack B. This factor is appealing as it allows the design of scaled current mirrors with minimal error.

\section{A. Multiplier Divider Design}

A translinear multiplier configuration is used, as presented by [9]. It is a conventional multiplier/divider circuit modified to produce smaller error between the output and input currents. This can be seen in Fig. 4 showing how its operation fits in well with the required operations of (11).

\section{B. Amplifier Bias Loop Design}

Following the multiplier operation, its output current is then scaled by the reference current and applied to the main signal amplifier using a servo current mirror. The simplified layout of this can be seen in Fig. 5. Each side of the current mirror

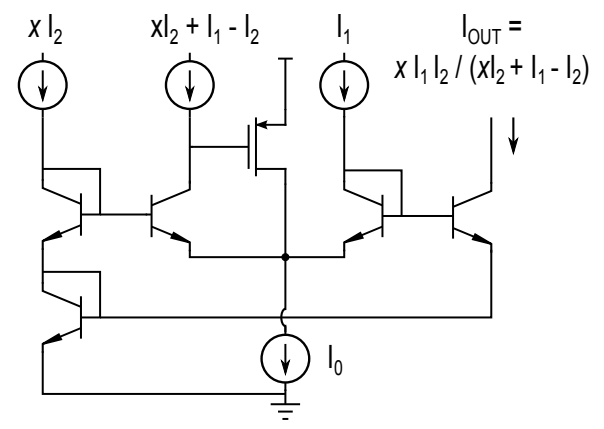

Fig. 4. Translinear multiplier used to perform algebraic operations required by the second circuit block.

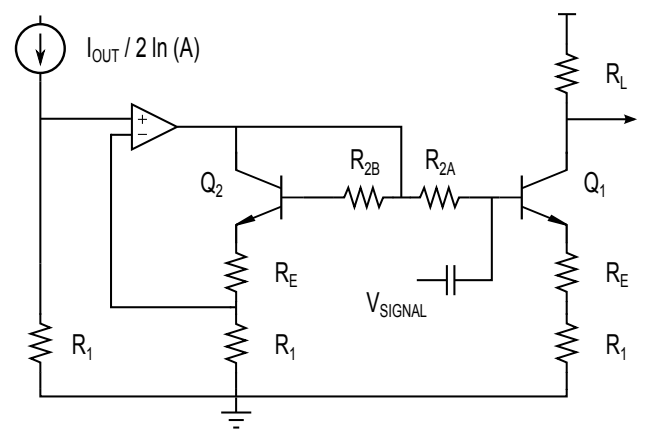

Fig. 5. Output bias loop used to set the bias current in the output transistor such that it operates at the third order null.

must be balanced in order to get an accurate bias current in the amplifier, hence the degeneration resistance $R_{1}$ is used on each side of the mirror, adding to the devices internal resistance value $\mathrm{RE}$ on each side. $R_{2 A}$ isolates the signal path from the bias section, and $R_{2 B}$ equalizes the voltage drop due to base currents. In order to further minimise error contributed by loss of base current from the reference, a base current compensation MOS transistor is used on the input side.

\section{ERROR ANALYSIS}

Error sensitivity is a major consideration in this design for two reasons. Firstly, the IM3 null is sharply defined so a small change in the target emitter voltage can lead to a large change in the IIP3 magnitude and secondly it is a moderately large circuit in which there is the potential for errors to accumulate.

\section{A. Multiplier Output Error}

Process variation simulations were undertaken based on industry typical corner values for this type of $0.5 \mu \mathrm{m} \mathrm{BiCMOS}$ technology. Resistor absolute values were varied over $\pm 20 \%$ and resistor matching simulations were made with $\pm 2 \%$ variation. Spice simulations show how these variations affect the bias current and the target emitter degeneration voltage. Nominal circuit values are chosen as $R_{1}=60 \Omega$, ambient temperature $=27^{\circ} \mathrm{C}$, and supply voltage $=3.3 \mathrm{~V}$. From simulation we see the equilibrium current in Stack A $\left(I_{1}\right)$ and Stack B $\left(I_{2}\right)$ are $304.0 \mu \mathrm{A}$ and $223.6 \mu \mathrm{A}$ respectively. These currents are used with (11) to calculate an ideal multiplier output current of $127.5 \mu \mathrm{A}$, our target current for all multiplier output error 


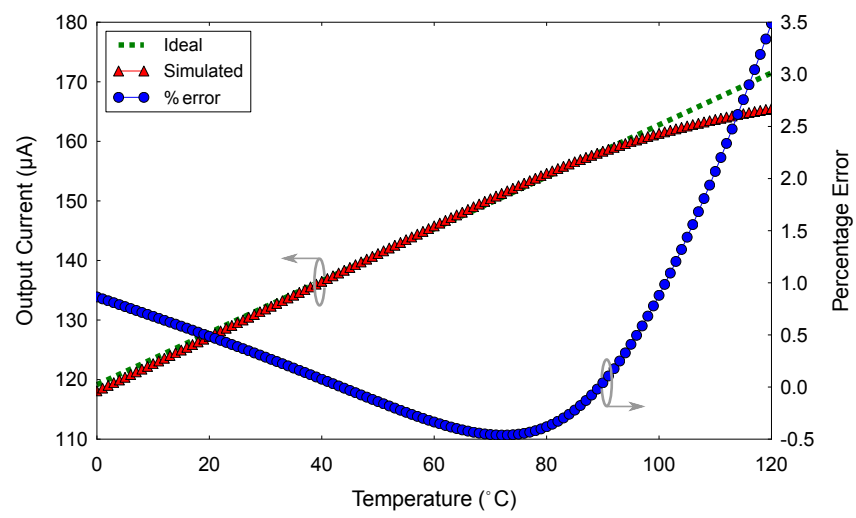

Fig. 6. Bias current variation vs. temperature variation compared with the ideal bias current, at the multiplier output.

calculations. Fig. 6 shows the variations of temperature as well as the resulting percentage error compared to the ideal calculated bias current, and it suggests the circuit is relatively unaffected by temperature variation. The data reveals that in the temperature range of $0-100^{\circ} \mathrm{C}$ the expected variation in bias current is $\leq 0.8 \%$. The error increases steadily at higher values than $100^{\circ} \mathrm{C}$, eg. $3.5 \%$ at $120^{\circ} \mathrm{C}$. Similar data for the supply voltage shows the worst case sensitivity is $\leq 3.1 \%$, obtained by varying the supply by $\pm 20 \%$.

\section{B. Signal Amplifier Bias Current Error}

Simulations were run for the variation in amplifier output bias current due to variations in $R_{1}$ and $R_{E}$ for the expected absolute and mismatch process errors. The results are summarised in Table 1. This shows the worst case variation from the ideal amplifier bias current is $\leq 2 \%$ in all cases.

\section{IM3 Nulling Error}

The primary goal of this work is to obtain a method of guaranteeing the bias of an amplifier at the null in 3rd order non-linearity, over process, supply variations, and temperature (PVT) and so the sensitivity of the complete system to IM3 is a critical measure. Fig. 7 shows the absolute and mismatch variations of $R_{1}$ and $R_{E}$ versus its effect on the IIP3 of the bias output current. From the nominal IIP3 value set by the nominal component values, these variations lead to a maximum IIP3 variation of $\pm 6.0 \mathrm{dBV}$, reflecting the bias current error of where the circuit sits in the IM3 null. Including temperature and supply variations of $20 \%$, the maximum IIP3 variation increases to approximately $\pm 9.5 \mathrm{dBV}$.

\section{CONCLUSION}

A bias circuit has been developed that sets a BJT amplifier in its natural IM3 null and can maintain this despite variations in temperature and in tolerances of the degeneration resistance. Furthermore, a technique has been developed using matched translinear stacks to extract the value of the transistors internal emitter resistance as fabricated on each die. This resistance extraction, combined with the PTAT currents generated, creates a reference bias that takes into account the total effective
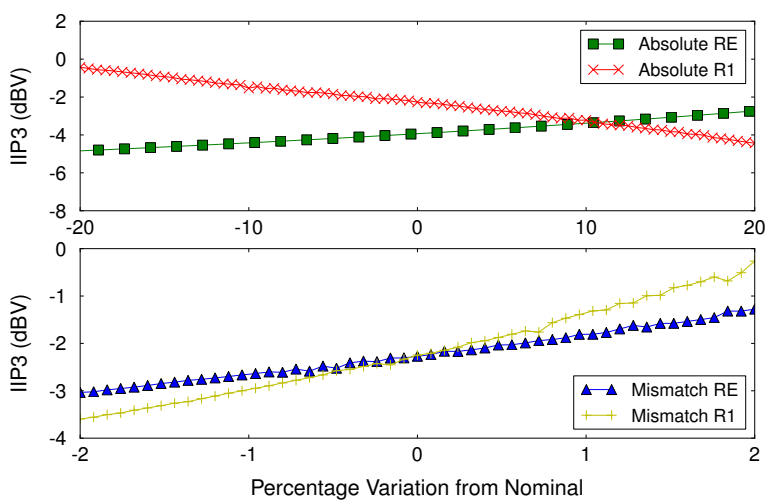

Fig. 7. Variation of absolute component values and mismatched component values vs. IIP3.

TABLE I

ERROR LIMITS IN BIAS CURRENT DUE TO COMPONENT VARIATION

\begin{tabular}{ccc}
\hline Variation type & Component & Error Limits \\
\hline \hline Absolute values (20\%) & $R_{1}$ & $-1.90 \%,-0.63 \%$ \\
& $R_{E}$ & $+0.42 \%,-1.95 \%$ \\
Mismatch values (2\%) & $R_{1}$ & $<0.1 \%,-1.97 \%$ \\
& $R_{E}$ & $-0.42 \%,-1.50 \%$ \\
\hline
\end{tabular}

degeneration resistance to fix the operating point at the IM3 null. The error limits of the extracted bias current show insensitivity to variation from key circuit components and with further work this error may be reduced. Temperature and supply voltage variations around the nominal design values are shown to have minimal effect and the IM3 of a demonstrator amplifier is maintained close to its ideal null.

\section{REFERENCES}

[1] W. Sansen, "Distortion in elementary transistor circuits," IEEE Transactions on Circuits and Systems II: Analog and Digital Signal Processing, vol. 46, no. 3, pp. 315-325, Mar. 1999.

[2] T. Balsom, J. Scott, and W. Redman-White, "Third Order Nulling Effect in Darlington Transistors," in Proceedings of the 18th Electronics New Zealand Conference, 2011.

[3] J. Reynolds, "Nonlinear Distortions and, Their Cancellation in Transistors," IEEE Transactions on Electron Devices, vol. 12, no. 11, pp. 595599, 1965.

[4] A. Helmy, K. Sharaf, and H. Ragai, "A simplified Analytical Model for Nonlinear Distortion in RF Bipolar Circuits," in Proc. 43rd IEEE Midwest Symp. on Circuits and Systems, 2000, pp. 2-5.

[5] G. K. Klimovitch, "On robust suppression of Third-Order Intermodulation Terms in Small-Signal Bipolar Amplifiers," Microwave Symposium Digest. IEEE MTT-S International, pp. 477-480, 2000.

[6] M. Hauser, "Large-Signal Electronically Variable Gain Techniques," Ph.D. dissertation, University of California, Berkeley, 1979.

[7] I. E. Opris, "Series Resistance Compensation in Translinear Circuits," IEEE Transactions on Circuits and Systems-1: Fundamental Theory and Applications, vol. 45, no. 1, pp. 91-94, 1998.

[8] J. Scott, "New Method to Measure Emitter Resistance of Heterojunction Bipolar Transistors," IEEE Transactions on Electron Devices, vol. 50, no. 9, pp. 1970-1973, 2003.

[9] M. Somdunyakanok, T. Pattanathadapong, and P. Prommee, "Accurate Tunable Current-mirror and its Applications," in International Symposium on Communications and Information Technologies, 2008, pp. 56-61. 